\title{
Artificial compliance inherent to the intrinsic cohesive zone models: criteria and application to planar meshes
}

\author{
BLAL Nawfal $^{1,2,3}$-DARIDON Loïc ${ }^{1,3}$ \\ MONERIE Yann ${ }^{2,3}$-PAGANO Stéphane ${ }^{1,3}$ \\ ${ }^{1}$ Laboratoire de Mécanique et Génie Civil, Montpellier \\ ${ }^{2}$ Institut de Radioprotection et de Sûreté Nucléaire, Cadarache \\ ${ }^{3}$ Laboratoire de Micromécanique et d'Intégrité des Structures \\ MIST Laboratory, IRSN-CNRS-Université Montpellier 2 \\ \{nawfal.blal, loic.daridon, stephane.pagano\}@univ-montp2.fr \\ yann.monerie@irsn.fr
}

\begin{abstract}
In this study, criteria on the artificial compliance due to intrinsic cohesive zone models are presented. The approach is based on a micromechanical model for a collection of cohesive zone models embedded between each mesh of a finite element-type discretization. The overall elastic behaviour of this cohesive volumetric medium is obtained using homogenization techniques and is given in a closed-form as function of bulk properties of the relevant material and mesh parameters (the mesh type and size). Practical criteria are obtained for the calibration of the cohesive stiffnesses bounding the additional compliance inherent to intrinsic cohesive zone models by lower value. For isotropic planar discretizations (e.g. Delaunay mesh), a rigorous bound is derived whereas convenient estimates are given for non-isotropic discretizations (e.g. regular mesh).
\end{abstract}

Keywords: Micromechanics; Damage; Cohesive Zone Models; Homogenization; Planar meshes

\section{Introduction}

Since the pioneering works of Dugdale (1960) and Barenblatt (1962), cohesive approaches had emerged as one of the most efficient methods used for numerical fracture mechanics. Their numerical simplicity as their capacity to simulate numerical fracture processes from crack initiation to overall failure without any ad hoc criteria are the most appealing features of these models. However, their numerical implementation exhibits a strong mesh sensitivity which is still an issue of concern: as shown in Tijssens et al. (2000), the fracture paths depend on the mesh geometry and size. Despite this path sensitivity, the authors show therein that the overall force-displacement response is not very sensitive to the mesh size and can be predicted with reasonable accuracy. Another aspect of this mesh sensitivity concerns the intrinsic Cohesive Zone Models (CZM), i.e. traction-separation laws with initial slope: incorporating cohesive zones between each couple of adjacent elements of a finite element-type discretization leads to an additional compliance since the density of the cohesive interfaces increases as the mesh is refined. This aspect can be illustrated on a simple 1D case (see Figure 1), where a linear elastic intrinsic CZM is inserted between two bulk elements. In that case, the equilibrium condition reads:

$$
\frac{F}{S}=E^{\mathrm{M}} \varepsilon=C_{\mathrm{N}} \llbracket \boldsymbol{u} \rrbracket
$$

where $F$ is the applied force, $\varepsilon$ the strain in the bulk elements (mesh size $L_{\text {mesh }}$, cross section $S$ ), $E^{\mathrm{M}}$ the Young's modulus used in the bulk element constitutive behaviour, $C_{N}$ the initial cohesive stiffness of the intrinsic model and $\llbracket \boldsymbol{u} \rrbracket$ is the displacement jump across the cohesive zone.The overall strain of this 
composite is:

$$
\widetilde{\varepsilon}=\frac{F / S}{E^{\mathrm{M}}}+\frac{F / S}{C_{\mathrm{N}} L_{\text {mesh }}} .
$$

Since the equilibrium condition requires $F / S=\widetilde{E} \widetilde{\varepsilon}$, where $\widetilde{E}$ denotes the apparent Young's modulus of the assemblage, one can deduce the normalized apparent Young's modulus:

$$
\frac{\widetilde{E}}{E^{\mathrm{M}}}=\frac{F / S}{E^{\mathrm{M} \widetilde{\varepsilon}}}=\frac{\xi}{1+\xi} \quad \text { with } \quad \xi=\frac{C_{\mathrm{N}} L_{\mathrm{mesh}}}{E^{\mathrm{M}}}
$$

According to this last equation, the ratio $\xi$ appears as the key adimentional parameter in controlling the mesh sensitivity of intrinsic CZMs. More precisely, the added compliance vanishes $\left(\widetilde{E} \rightarrow E^{\mathrm{M}}\right)$ when $\xi \rightarrow+\infty$, see Figure 1 (b). In other words, the overall elastic behaviour is not affected by the introduction of intrinsic CZMs between bulk elements and therefore the mesh sensitivity effect vanishes.

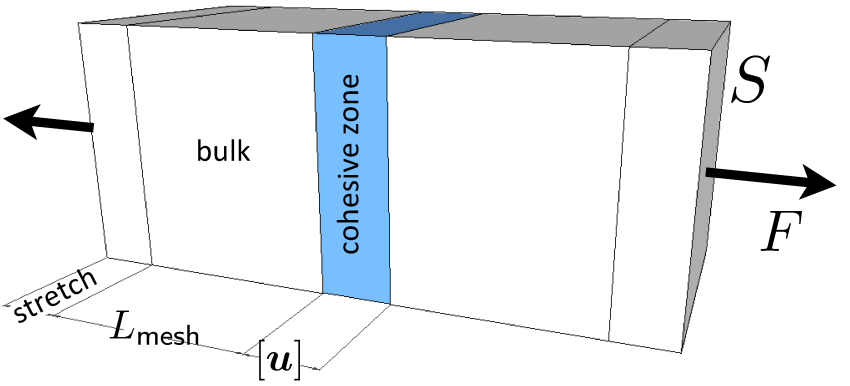

(a)

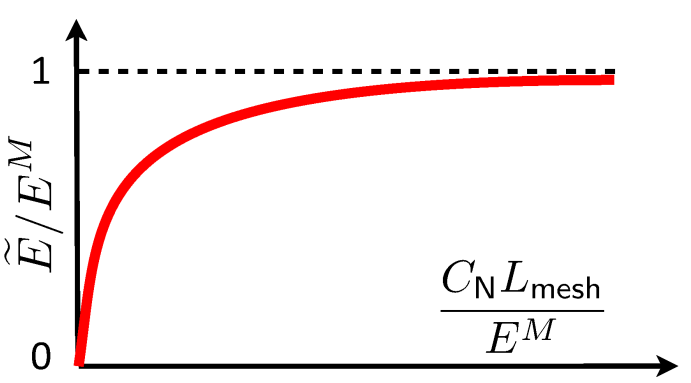

(b)

Figure 1: Illustration of the relationship between apparent Young's modulus and mesh size in 1D-case: (a) a representative part of 1D finite element mesh with embedded cohesive zone model, (b) overall Young's modulus normalized by bulk modulus vs mesh size.

Following the same type of ideas, various authors have proposed semi-empirical bound for the ratio $\xi$ in order to define 'invisible' CZMs at the scale of a structure. Equation (3) results from similar mechanical considerations in the work of Turon et al. (2007) concerning the numerical simulation of delamination: taking $\xi$ larger than $50(\xi>50)$ ensures that the apparent loss of stiffness is less than $2 \%$, hence the composite is not affected by the presence of cohesive interfaces. Performing numerical tension and shear tests, Espinosa and Zavattieri (2003) have noticed that the elastic wave speeds remain unchanged across a cohesive line between two elastic and isotropic media when $\xi \geq 10$. Estimating the added compliance for cross-triangle elements arranged in quadrilateral pattern submitted to uniaxial tension, biaxial uniform tension and pure shear, Tomar et al. (2004) obtain $\left(\nu^{M}\right.$ being the Poisson ratio of the bulk material):

$$
\xi \gg \alpha \frac{\sqrt{2}+1}{1-\nu^{M}}
$$

with $\alpha=1$ for plane stress and $\alpha=1 /\left(1-\left(\nu^{M}\right)^{2}\right)$ for plane strain. The aim of this work is to forth fully generalize these criteria to three dimensional situations and to any type of loadings.

\section{Micromechanical model}

We focus in the sequel on cohesive-volumetric finite element approach with intrinsic cohesive zone model: each volumetric element of a standard finite element discretization is connected to each couple of adjacent elements using intrinsic CZMs as boundary conditions. Recent developments suggest to replace the cohesive-volumetric approach with the coupling between CZMs and X-FEM, e.g. Benvenuti (2008), but this cumbersome technic is not widely spread in commercial codes. Moreover, the 
cohesive-volumetric approach can be used with extrinsic cohesive models, i.e. initially rigid or 'shifted' models (Hille et al., 2004). But most of the time, loading-unloading situations lead to elastic reload of the cohesive zones and the intrinsic case should anyway be considered. Intrinsic cohesive zone models embedded within a standard finite element discretization is thus investigated in the following as a reference case.

\subsection{Cohesive discretization as a "matrix-inclusion" composite}

Following Blal et al. (2011), the main idea is to replace a cohesive-volumetric finite element discretization by a continuous matrix containing penny-shaped cohesive inclusions (Figure 2). The matrix has

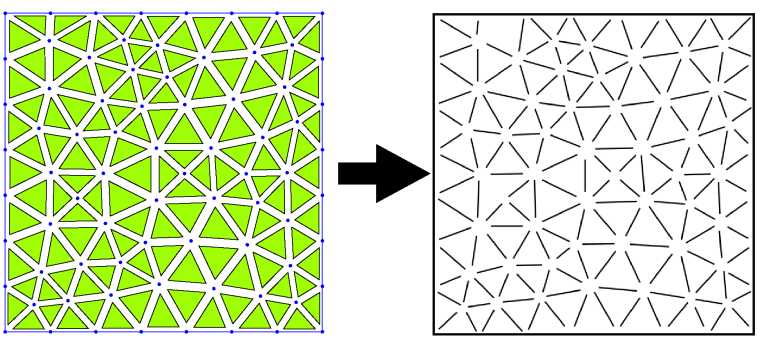

(a)

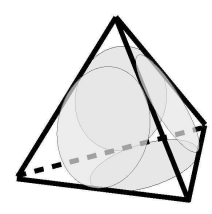

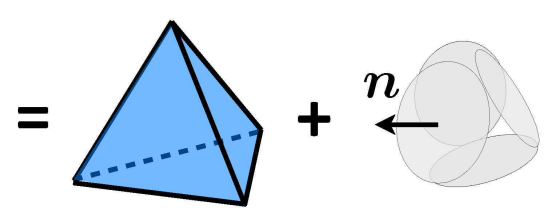

(b)

Figure 2: Principle of the approach: a cohesive-volumic finite element mesh is replaced by a continuous matrix corresponding to bulk elements and a collection of penny-shaped cohesive inclusions corresponding to the edges of the underlying mesh; (a) 2-D illustration, (b) 3-D illustration.

the same behaviour as the bulk finite element behaviour whereas the penny-shaped inclusions have a cohesive behaviour defined by a traction-separation law. The spatial distribution of the cohesive inclusions corresponds to those of the edges of the underlying mesh and has the same density, denoted by $Z$. In particular, in the case of a statistical isotropic mesh, e.g. a Delaunay-type mesh, the inclusions are randomly distributed in space and in orientation. The density $Z$ corresponds to the specific 'surface' of the interface between the meshes: in two dimensions, $Z$ is equal to the ratio of the total length of the edges to the area of the meshed surface; in three dimensions, $Z$ is equal to the ratio of the total area of the edges to the volume of the meshed body. Moreover, the next assumption is made concerning the shape of the inclusions:

Hypothesis 1. The inclusions are assumed to be penny-shaped (Figure 2). In two dimensions, the straight edges of the mesh are thus replaced by zero thickness whiskers and this assumption has no consequence. In three dimensions, the polygonal edges are replaced by disks (with the same spatial density): the two situations are admittedly close to each other but are different when considered rigorously.

\subsection{Phases properties}

In the sequel, we restrict our attention to linear elastic behaviours. Moreover, the matrix phase is considered as isotropic and its constitutive relation reads:

$$
\boldsymbol{\sigma}=\mathbb{C}^{\mathrm{M}}: \boldsymbol{\varepsilon} \quad \text { with } \quad \mathbb{C}^{\mathrm{M}}=3 k^{M} \mathbb{J}+2 \mu^{M} \mathbb{K},
$$

where $\boldsymbol{\sigma}$ (resp. $\varepsilon$ ) is the stress field (resp. strain), $\mathbb{C}^{\mathrm{M}}$ is a fourth order stiffness tensor, $k^{\mathrm{M}}$ and $\mu^{M}$ are the bulk and the shear modulus respectively. The symmetric tensors $\mathbb{J}$ and $\mathbb{K}$ define the generic basis of the fourth order isotropic symmetric tensors:

$$
\mathbb{J}=\frac{1}{3} \boldsymbol{i} \otimes \boldsymbol{i} \quad \text { and } \quad \mathbb{K}=\mathbb{I}-\mathbb{J} \quad \text { with } \quad 2 \mathbb{I}_{i j k l}=\left(\boldsymbol{i}_{i k} \boldsymbol{i}_{j l}+\boldsymbol{i}_{i l} \boldsymbol{i}_{j k}\right)
$$


where $i$ is the second order identity tensor. The previous constitutive law (5) can be written in a matrix form as follows:

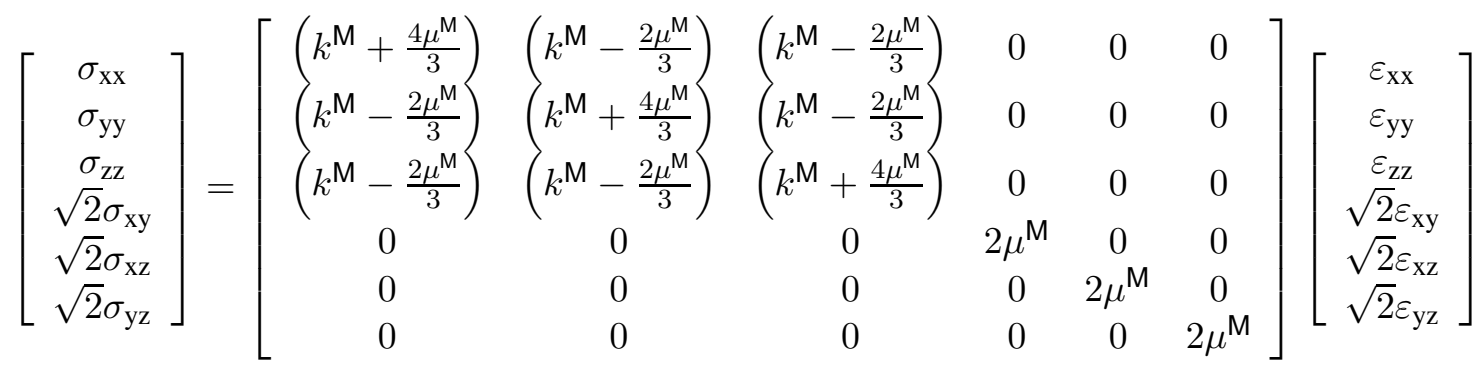

The cohesive traction-separation law corresponds to a linear relation between the cohesive stress vector $\mathbf{R}^{\text {coh }}$ and the opening displacement vector $\llbracket \boldsymbol{u} \rrbracket=\boldsymbol{u}^{+}-\boldsymbol{u}^{-}$where $\boldsymbol{u}^{+}$(resp. $\boldsymbol{u}^{-}$) is the displacement of the points located on the top (resp. bottom) side of the interface. In the local normal-tangent basis oriented by the normal vector $\boldsymbol{n}$, the jump displacement vector can be decomposed to a normal, $u_{\mathrm{N}}$, and a tangential, $\boldsymbol{u}_{\top}$, component, so that: $\llbracket \boldsymbol{u} \rrbracket=u_{\mathrm{N}} \boldsymbol{n}+\boldsymbol{u}_{\top}$. The cohesive constitutive relation reads:

$$
\mathbf{R}^{\text {coh }}=\boldsymbol{K} \cdot \llbracket \boldsymbol{u} \rrbracket \quad \text { with } \quad \boldsymbol{K}=C_{\mathrm{N}} \boldsymbol{n} \otimes \boldsymbol{n}+C_{\mathrm{T}}(\boldsymbol{i}-\boldsymbol{n} \otimes \boldsymbol{n}),
$$

where $C_{\mathrm{N}}$ (resp. $C_{\mathrm{T}}$ ) is the normal (resp. tangential) initial 'stiffness' of the cohesive law. The cohesive stress tensor is given thus combining (8) and the relationship $\mathbf{R}^{\mathrm{coh}}=\boldsymbol{\sigma}^{\mathrm{coh}} \cdot \boldsymbol{n}$ as proposed in Acary and Monerie (2006):

$$
\boldsymbol{\sigma}^{\mathrm{coh}}=\left(C_{\mathrm{N}} \mathbb{E}_{l}+C_{\mathrm{\top}} \mathbb{K}_{l}\right):\left(\llbracket \boldsymbol{u} \rrbracket \otimes_{s} \boldsymbol{n}\right),
$$

where $\otimes_{s}$ is the symmetric dyadic product $\left(2 \boldsymbol{a} \otimes_{s} \boldsymbol{b}=a_{i} b_{j}+a_{j} b_{i}\right.$ for any vector $\boldsymbol{a}$ and $\left.\boldsymbol{b}\right)$ and $\mathbb{E}_{l}, \mathbb{K}_{l}$ are two components of the fourth order transversely isotropic and symmetric tensors frame:

$$
\mathbb{E}_{l}=\boldsymbol{n} \otimes \boldsymbol{n} \otimes \boldsymbol{n} \otimes \boldsymbol{n} \quad \text { and } \quad \mathbb{K}_{l}=2\left(\boldsymbol{j}_{\boldsymbol{s}} \otimes \boldsymbol{j}_{\boldsymbol{s}}+\boldsymbol{j}_{\boldsymbol{t}} \otimes \boldsymbol{j}_{\boldsymbol{t}}\right)
$$

with $j_{s}=n \otimes_{s} s$ and $j_{t}=n \otimes_{s} \boldsymbol{t}$, where $s$ and $\boldsymbol{t}$ are two orthogonal vectors defining the transversal plane ( $\boldsymbol{n}, \boldsymbol{t}, \boldsymbol{s}$ define the local orthogonal basis of the cohesive inclusion). Defining the cohesive 'strain' with the help of a small length $e$ (Michel et al., 1994):

$$
\varepsilon^{\mathrm{coh}}=\frac{\llbracket \boldsymbol{u} \rrbracket \otimes_{s} \boldsymbol{n}}{e}
$$

a fourth order stiffness tensor $\mathbb{C}^{\text {coh }}$ can be deduced for the cohesive phase. The constitutive relation thus reads for the inclusions:

$$
\sigma^{\text {coh }}=\mathbb{C}^{\text {coh }}: \varepsilon^{\text {coh }} \quad \text { with } \quad \mathbb{C}^{\text {coh }}=e\left(C_{\mathrm{N}} \mathbb{E}_{l}+C_{\top} \mathbb{K}_{l}\right) .
$$

According to the local frame $(\boldsymbol{n}, \boldsymbol{t}, \boldsymbol{s})$, the cohesive governing equation (12) reads in a reduced form:

$$
\left[\begin{array}{c}
\sigma_{\mathrm{nn}} \\
\sigma_{\mathrm{tt}} \\
\sigma_{\mathrm{ss}} \\
\sqrt{2} \sigma_{\mathrm{nt}} \\
\sqrt{2} \sigma_{\mathrm{ns}} \\
\sqrt{2} \sigma_{\mathrm{st}}
\end{array}\right]=e\left[\begin{array}{cccccc}
C_{\mathrm{N}} & 0 & 0 & 0 & 0 & 0 \\
0 & 0 & 0 & 0 & 0 & 0 \\
0 & 0 & 0 & 0 & 0 & 0 \\
0 & 0 & 0 & C_{\mathrm{T}} & 0 & 0 \\
0 & 0 & 0 & 0 & C_{\mathrm{T}} & 0 \\
0 & 0 & 0 & 0 & 0 & 0
\end{array}\right]\left[\begin{array}{c}
\varepsilon_{\mathrm{nn}} \\
\varepsilon_{\mathrm{tt}} \\
\varepsilon_{\mathrm{ss}} \\
\sqrt{2} \varepsilon_{\mathrm{nt}} \\
\sqrt{2} \varepsilon_{\mathrm{ns}} \\
\sqrt{2} \varepsilon_{\mathrm{st}}
\end{array}\right]
$$

The small length $e$, that should tend to zero, is a fictitious thickness associated to the cohesive zone. Since oblate ellipsoids tend to penny-shaped inclusions when their thickness tends to zero, the cohesive inclusions are now considered as oblate ellipsoids and their volume fraction $f$ is the product of the density $Z$ (inversely proportional to a length, namely the mesh size $L_{\text {mesh }}$ ) and of the fictitious thickness $e$ :

$$
f=e Z
$$

where the cylindrical hypothesis of inclusions instead of oblate one has no consequence when $e \rightarrow 0$. 


\subsection{The overall homogeneous behaviour: Hashin-Shtrickman estimate}

A Hashin-Shtrikman estimate (Hashin and Shtrikman, 1963) is used in order to establish an analytical expression of the overall elastic stiffness, denoted by $\mathbb{C}^{\text {hom }}$. The considered matrix-inclusion composite (Figure 2) is composed of an isotropic elastic matrix (volume fraction $1-f$ ) following the constitutive relation (5) and of a collection of transversely isotropic oblate ellipsoids (volume fraction $f$ ) with the largest semi-axis $a$ varying from $A^{-}$to $A^{+}$, with the same aspect ratio $w=b / a \ll 1$ ( $b$ is the smallest semi-axis, i.e. along the dimension of the ellipsoids along the symmetry axis) and following the constitutive relation (8). The orientation of these ellipsoids is characterized by the direction of their symmetry axis, i.e. a vector $\boldsymbol{n}$ of the unit sphere $S$ of $\mathbb{R}^{3}$ (measure $4 \pi$ ). This collection of inclusions is splitted into $N-1$ families $r(r=2, \ldots, N)$, the $r$-th family being defined by a radius included in $(a, a+d a)$ and an orientation lying within the elementary solid angle $d s$ centered on $\boldsymbol{n}$. Defining the inclusion density $\phi(a, \boldsymbol{n})$ such that $\phi(a, \boldsymbol{n}) d a d s$ is the number of inclusions of the $r$-th family in a unit volume, the volume fraction by unit volume $c_{r}$ of the $r$-th family reads:

$$
\begin{gathered}
c_{r}=\phi(a, \boldsymbol{n}) \frac{4 \pi}{3} a^{2} b d a d s=\phi(a, \boldsymbol{n}) \frac{4 \pi}{3} a^{3} w d a d s \quad(r=2, \ldots, N), \\
\text { with } f=\sum_{r=2}^{N} c_{r}=\int_{S} \int_{A^{-}}^{A^{+}} \phi(a, \boldsymbol{n}) \frac{4 \pi}{3} a^{3} w d a d s .
\end{gathered}
$$

For this situation, the following generalization of the Hashin-Shtrikman's result is considered (Willis, 1977):

$$
\begin{aligned}
& \mathbb{C}^{\mathrm{HS}}\left(\mathbb{C}^{0}\right)= \\
& {\left[(1-f) \mathbb{C}^{\mathrm{M}}:\left(\mathbb{I}+\mathbb{P}:\left(\mathbb{C}^{\mathrm{M}}-\mathbb{C}^{0}\right)\right)^{-1}+\sum_{r=2}^{N} c_{r} \mathbb{C}_{r}^{\mathrm{coh}}:\left(\mathbb{I}+\mathbb{P}:\left(\mathbb{C}_{r}^{\mathrm{coh}}-\mathbb{C}^{0}\right)\right)^{-1}\right]} \\
& :\left[(1-f)\left(\mathbb{I}+\mathbb{P}:\left(\mathbb{C}^{\mathrm{M}}-\mathbb{C}^{0}\right)\right)^{-1}+\sum_{r=2}^{N} c_{r}\left(\mathbb{I}+\mathbb{P}:\left(\mathbb{C}_{r}^{\mathrm{coh}}-\mathbb{C}^{0}\right)\right)^{-1}\right]^{-1}
\end{aligned}
$$

where $\mathbb{C}^{0}$ (resp. $\mathbb{C}_{r}^{\text {coh }}$ ) is the fourth order stiffness tensor of a reference medium (resp. of the $r$-th family of inclusions) and $\mathbb{P}$ is the Hill polarization tensor which characterizes the stress that the infinite surrounding reference medium applies on a single ellipsoid having a homogeneous eigenstrain. The Hill tensor $\mathbb{P}$ depends on the shape of the inclusion $w$, on its orientation $\boldsymbol{n}$ and on the stiffness tensor of the reference medium $\mathbb{C}^{0}$. Following Willis (1977), this reference medium is a homogeneous material such that the constitutive relations (5) and (12) are replaced respectively with $\sigma=\mathbb{C}^{0}: \varepsilon+\tau$ and $\sigma^{\text {coh }}=\mathbb{C}^{0}$ : $\varepsilon^{\text {coh }}+\tau^{\text {coh }}$ where the polarizations $\tau$ and $\tau^{\text {coh }}$ reads: $\tau=\left(\mathbb{C}-\mathbb{C}^{0}\right): \varepsilon$ and $\tau^{\text {coh }}=\left(\mathbb{C}^{\text {coh }}-\mathbb{C}^{0}\right): \varepsilon^{\text {coh }}$. Using the Hill influence tensor $\mathbb{C}^{*}=\mathbb{P}^{-1}-\mathbb{C}^{0}$, and introducing the two fourth order tensors $\mathbb{V}_{1}=$ $\left(\mathbb{C}^{*}+\mathbb{C}^{\mathrm{M}}\right)^{-1}$ and $\mathbb{V}_{r}=\left(\mathbb{C}^{*}+\mathbb{C}_{r}^{\mathrm{coh}}\right)^{-1}$, one obtains:

$$
\begin{aligned}
\mathbb{I}+\mathbb{P}:\left(\mathbb{C}^{\mathrm{M}}-\mathbb{C}^{0}\right) & =\mathbb{I}+\left(\mathbb{C}^{*}+\mathbb{C}^{0}\right)^{-1}:\left(\mathbb{C}^{\mathrm{M}}-\mathbb{C}^{0}\right) \\
& =\left(\mathbb{C}^{*}+\mathbb{C}^{0}\right)^{-1}:\left(\mathbb{C}^{*}+\mathbb{C}^{\mathrm{M}}\right) \\
& =\left(\mathbb{C}^{*}+\mathbb{C}^{0}\right)^{-1}: \mathbb{V}_{1}^{-1}
\end{aligned}
$$

and the same relation holds for

$$
\mathbb{I}+\mathbb{P}:\left(\mathbb{C}_{r}^{\text {coh }}-\mathbb{C}^{0}\right)=\left(\mathbb{C}^{*}+\mathbb{C}^{0}\right)^{-1}: \mathbb{V}_{r}^{-1} .
$$

Combining these two last relations and simplifying by $\left(\mathbb{C}^{*}+\mathbb{C}^{0}\right)$, equation $(15)$ is rearranged as:

$$
\mathbb{C}^{\mathrm{HS}}\left(\mathbb{C}^{0}\right)=\left[(1-f) \mathbb{C}^{\mathrm{M}}: \mathbb{V}_{1}+\sum_{r=2}^{N} c_{r} \mathbb{C}_{r}^{\mathrm{coh}}: \mathbb{V}_{r}\right]:\left[(1-f) \mathbb{V}_{1}+\sum_{r=2}^{N} c_{r} \mathbb{V}_{r}\right]^{-1}
$$


Introducing $\mathbb{T}_{r}=\mathbb{V}_{r}: \mathbb{V}_{1}^{-1}$ and remarking that the cohesive stiffness depends only on the orientation $\boldsymbol{n}$, i.e. $\mathbb{C}_{r}^{\text {coh }}=\mathbb{C}^{\text {coh }}(\boldsymbol{n})$, and that tensor $\mathbb{T}_{r}$ depends only on the aspect ratio $w$ and the direction $\boldsymbol{n}$, i.e.

$$
\mathbb{T}_{r}=\mathbb{T}(w, \boldsymbol{n})=\left(\mathbb{C}^{*}(w, \boldsymbol{n})+\mathbb{C}^{\mathrm{coh}}(\boldsymbol{n})\right)^{-1}:\left(\mathbb{C}^{*}(w, \boldsymbol{n})+\mathbb{C}^{\mathrm{M}}\right)
$$

the estimate (16) is rewritten with the help of the density $\phi(a, \boldsymbol{n})$, equation (14) and an integration over the radius and the orientation:

$$
\begin{aligned}
\mathbb{C}^{\mathrm{HS}}\left(\mathbb{C}^{0}\right)= & {\left[(1-f) \mathbb{C}^{\mathrm{M}}+\int_{S} \int_{A^{-}}^{A^{+}} \phi(a, \boldsymbol{n}) \frac{4 \pi}{3} a^{3} w \mathbb{C}^{\mathrm{coh}}(\boldsymbol{n}): \mathbb{T}(w, \boldsymbol{n}) d a d s\right] } \\
& :\left[(1-f) \mathbb{I}+\int_{S} \int_{A^{-}}^{A^{+}} \phi(a, \boldsymbol{n}) \frac{4 \pi}{3} a^{3} w \mathbb{T}(w, \boldsymbol{n}) d a d s\right]^{-1} .
\end{aligned}
$$

Since $w$ is assumed to be constant and $\mathbb{C}^{\operatorname{coh}}(\boldsymbol{n})$ and $\mathbb{T}(w, \boldsymbol{n})$ do not depend on the radius $a$, integrating over $a$ leads to:

$$
\begin{array}{r}
\mathbb{C}^{\mathrm{HS}}\left(\mathbb{C}^{0}\right)=\left[(1-f) \mathbb{C}^{\mathrm{M}}+w \int_{S} \psi(\boldsymbol{n}) \mathbb{C}^{\mathrm{coh}}(\boldsymbol{n}): \mathbb{T}(w, \boldsymbol{n}) d s\right]: \\
{\left[(1-f) \mathbb{I}+w \int_{S} \psi(\boldsymbol{n}) \mathbb{T}(w, \boldsymbol{n}) d s\right]^{-1}}
\end{array}
$$

where

$$
\psi(\boldsymbol{n})=\int_{A^{-}}^{A^{+}} \frac{4}{3} \pi a^{3} \phi(a, \boldsymbol{n}) d a
$$

If the underlying mesh contains specific orientations (e.g. anisotropic meshes), they have to be taken into account through the density $\psi(\boldsymbol{n})$. For simplicity, isotropic meshes (e.g. Delaunay-type meshes) are considered in the next section.

\subsection{Lower bound for isotropic meshes}

We now specialize (19) for the case of an isotropic microstructure: both matrix and the mesh distribution are isotropic. Hence, the orientations of inclusions are assumed to have an equi-probability property (randomly oriented inclusions corresponding to isotropic overall meshes), the density $\psi(\boldsymbol{n})$ is reduced to $f /(4 \pi w)$ and the estimate (19) becomes $(f=e Z)$ :

$$
\begin{aligned}
\mathbb{C}^{\mathrm{HS}}\left(\mathbb{C}^{0}\right) & =\left[\frac{f}{4 \pi} \int_{S} \mathbb{C}^{\mathrm{coh}}: \mathbb{T}(w, \boldsymbol{n}) d s+(1-f) \mathbb{C}^{\mathrm{M}}\right]:\left[\frac{f}{4 \pi} \int_{S} \mathbb{T}(w, \boldsymbol{n}) d s+(1-f) \mathbb{I}\right]^{-1} \\
& =\left[e Z\left\langle\mathbb{C}^{\mathrm{coh}}: \mathbb{T}\right\rangle_{\circlearrowleft}+(1-e Z) \mathbb{C}^{\mathrm{M}}\right]:\left[e Z\langle\mathbb{T}\rangle_{\circlearrowleft}+(1-e Z) \mathbb{I}\right]^{-1}
\end{aligned}
$$

where $\langle.\rangle_{\circlearrowleft}$ indicates the average over all orientations. Following Gatt et al. (2005), this orientational average is equivalent to a projection over the isotropic frame for the fourth order symmetric tensors:

$$
\langle\mathbb{D}\rangle_{\circlearrowleft}=\frac{\mathbb{J}:: \mathbb{D}}{\mathbb{J}:: \mathbb{J}} \mathbb{J}+\frac{\mathbb{K}:: \mathbb{D}}{\mathbb{K}:: \mathbb{K}} \mathbb{K} \text { for any fourth order tensor } \mathbb{D},
$$

and the overall stiffness tensor is obtained after the passage to the limit: $\mathbb{C}^{\text {hom }}=\lim _{e \rightarrow 0} \mathbb{C}^{\mathrm{HS}}\left(\mathbb{C}^{0}\right)$.

At this stage, according to the choice of the reference medium $\mathbb{C}^{0}$, different bounds and estimates can be derived. Since, the normal and the tangential cohesive stiffnesses $C_{\mathrm{N}}$ and $C_{\mathrm{T}}$ have bounded values, the cohesive stiffness tensor $\mathbb{C}^{\text {coh }}$ tends to zero when the small length $e$ tends to zero (see equation (12)). At the limit $e \rightarrow 0, \mathbb{C}^{\text {coh }}$ is thus always smaller in a quadratic sense than the matrix elastic tensor $\mathbb{C}^{\mathrm{M}}$. The choice of the reference medium $\mathbb{C}^{0}=\mathbb{C}^{\mathrm{M}}$ corresponds to the Hashin-shtrickman upper bound (or the Mori-Tanake estimate) and gives the trivial result $\mathbb{C}^{\text {hom }}=\mathbb{C}^{\mathrm{M}}$ at the limit $e \rightarrow 0$. The same trivial result 
is obtained for the self-constitent scheme $\left(\mathbb{C}^{0}=\mathbb{C}^{\text {hom }}\right)$. Thus, by focusing on the case $\mathbb{C}^{0}=\mathbb{C}^{\text {coh }}$, i.e. using the most compliant phase as a reference medium the Hashin-Shtrickman lower bound is defined $\mathbb{C}^{\mathrm{HS}-}=\mathbb{C}^{\mathrm{HS}}\left(\mathbb{C}^{\mathrm{coh}}\right)$ :

$$
\begin{aligned}
\mathbb{C}^{\text {hom }} & =\lim _{e \rightarrow 0} \mathbb{C}^{\mathrm{HS}-} \\
& =\lim _{e \rightarrow 0}\left[e Z\left\langle\mathbb{C}^{\mathrm{coh}}: \mathbb{T}\right\rangle_{\circlearrowleft}+(1-e Z) \mathbb{C}^{\mathrm{M}}\right]:\left[e Z\langle\mathbb{T}\rangle_{\circlearrowleft}+(1-e Z) \mathbb{I}\right]^{-1}
\end{aligned}
$$

where the tensor $\mathbb{T}$ is reduced in this case to:

$$
\mathbb{T}=\mathbb{T}\left(\mathbb{C}^{\mathrm{coh}}\right)=\mathbb{I}+\mathbb{P}\left(\mathbb{C}^{\mathrm{coh}}\right):\left(\mathbb{C}^{\mathrm{M}}-\mathbb{C}^{\mathrm{coh}}\right) .
$$

The explicit analytical expression of the fourth order tensor $\mathbb{C}^{\text {hom }}$ can be conveniently obtained by representing the different tensors in terms of the components of the generic basis of transversely isotropic symmetric and fourth order tensors. Any transversely isotropic symmetric and fourth order tensors $\mathbb{C}$ can be linearly decomposed as follows (see Bornert et al. (2001) for example):

$$
\mathbb{C}=C_{1} \mathbb{E}_{l}+C_{2} \mathbb{J}_{t}+C_{3}\left(\mathbb{F}+{ }^{\top} \mathbb{F}\right)+C_{5} \mathbb{K}_{t}+C_{6} \mathbb{K}_{l},
$$

where $\mathbb{E}_{l}$ and $\mathbb{K}_{l}$ are previously introduced in (10) and the other components of the transversely isotropic tensor frame are given by:

$$
\begin{aligned}
& \boldsymbol{i}_{t}=\boldsymbol{i}-\boldsymbol{n} \otimes \boldsymbol{n} \quad \mathbb{J}_{t}=\frac{1}{2} \boldsymbol{i}_{t} \otimes \boldsymbol{i}_{t}, \\
& \mathbb{F}=\frac{1}{\sqrt{2}} \boldsymbol{i}_{t} \otimes \boldsymbol{n} \otimes \boldsymbol{n} \quad \mathbb{K}_{t}=\mathbb{I}_{t}-\mathbb{J}_{t} .
\end{aligned}
$$

with $\mathbb{I}_{t}$ being the fourth order transversal identity and $\boldsymbol{n}$ the main axis of the transversely isotropic medium (here $\boldsymbol{n}$ coincides with the outward normal of the penny-shaped cohesive inclusions). It follows that the sum and the product of two tensors $\mathbb{C}$ and $\mathbb{Q}$ are immediately given by:

$$
\begin{array}{r}
\mathbb{C}+\mathbb{Q}=\left(C_{1}+Q_{1}\right) \mathbb{E}_{l}+\left(C_{2}+Q_{2}\right) \mathbb{J}_{t}+\left(C_{3}+Q_{3}\right)\left(\mathbb{F}+{ }^{\top} \mathbb{F}\right)+\left(C_{5}+Q_{5}\right) \mathbb{K}_{t} \\
+\left(C_{6}+Q_{6}\right) \mathbb{K}_{l}, \\
\mathbb{C}: \mathbb{Q}=\left(C_{1} Q_{1}+C_{3} Q_{3}\right) \mathbb{E}_{l}+\left(C_{2} Q_{2}+C_{3} Q_{3}\right) \mathbb{J}_{t}+\left(C_{1} Q_{3}+C_{3} Q_{2}\right) \mathbb{F} \\
+\left(C_{3} Q_{1}+C_{2} Q_{3}\right)^{\top} \mathbb{F}+C_{5} Q_{5} \mathbb{K}_{t}+C_{6} Q_{6} \mathbb{K}_{l} .
\end{array}
$$

Moreover, the inverse of a tensor $\mathbb{C}$ can be obtained using simple algebraic manipulations:

$$
\mathbb{C}^{-1}=\frac{C_{1}}{\Delta} \mathbb{E}_{l}+\frac{C_{2}}{\Delta} \mathbb{J}_{t}+\frac{-C_{3}}{\Delta}\left(\mathbb{F}+{ }^{\top} \mathbb{F}\right)+\frac{1}{C_{5}} \mathbb{K}_{t}+\frac{1}{C_{6}} \mathbb{K}_{l}
$$

where $\Delta=C_{1} C_{2}-C_{3}^{2}$.

As first step, the cohesive stiffness tensor is re-written in the transversely isotropic frame:

$$
\mathbb{C}^{\text {coh }}=e\left(C_{\mathrm{N}} \mathbb{E}_{l}+C_{\mathrm{A}} \mathbb{J}_{t}+C_{\mathrm{B}}\left(\mathbb{F}+{ }^{\top} \mathbb{F}\right)+C_{\mathrm{C}} \mathbb{K}_{t}+C_{\mathrm{T}} \mathbb{K}_{l}\right),
$$

where the coefficients, $C_{\mathrm{A}}, C_{\mathrm{B}}$ and $C_{\mathrm{C}}$ do not have any physical sense and are introduced in order to calculate the inverse tensors occurring in the expression of the Hashin-Shtrikman estimate; indeed, these coefficients will naturally disappear when $e$ tends to zero. The expression of the polarization tensor $\mathbb{P}$ is then derived for penny-shaped inclusions (radius $R$ ) with the cohesive inclusions (28) as the reference medium using the method proposed in Sevostianov et al. (2005):

$$
\begin{aligned}
\mathbb{P}(e, \boldsymbol{n}) & =\frac{1}{C_{\mathrm{N}}} e \mathbb{E}_{l}+\frac{3 \pi}{4 \sqrt{2} C_{\mathrm{N}} R}\left(\mathbb{F}+{ }^{\top} \mathbb{F}\right)-\frac{\pi}{C_{\mathrm{\top}} R}\left(\mathbb{J}_{t}+\mathbb{K}_{t}\right) \\
& +\frac{1}{C_{\mathrm{\top}} e} \mathbb{K}_{t}+\frac{C_{\mathrm{A}}+2 C_{\mathrm{C}}}{4 C_{\mathrm{\top}}^{2} R} \pi \mathbb{K}_{l} .
\end{aligned}
$$


The Projection of the matrix stiffness tensor $\mathbb{C}^{\mathrm{M}}$ in the transversely isotropic frame:

$$
\begin{aligned}
\mathbb{C}^{\mathrm{M}} & =\left[k^{\mathrm{M}}+\frac{4 \mu^{\mathrm{M}}}{3}\right] \mathbb{E}_{l}+\left[\sqrt{2}\left(k^{\mathrm{M}}-\frac{2 \mu^{\mathrm{M}}}{3}\right)\right]\left(\mathbb{F}+{ }^{\mathrm{T}} \mathbb{F}\right) \\
& +\left[2 k^{\mathrm{M}}+\frac{2 \mu^{\mathrm{M}}}{3}\right] \mathbb{J}_{t}+2 \mu^{\mathrm{M}}\left(\mathbb{K}_{t}+\mathbb{K}_{l}\right),
\end{aligned}
$$

and the substitution of equations (5), (28) and (29) into (23) leads to:

$$
\begin{aligned}
\mathbb{T} & =\left[\frac{9 e \pi+12 R}{12 C_{\mathrm{N}} e R} k^{\mathrm{M}}+\left(\frac{4}{3 C_{\mathrm{N}} e}-\frac{\pi}{2 C_{\mathrm{N}} R}\right) \mu^{\mathrm{M}}\right] \mathbb{E}_{l} \\
& +\left[-\frac{3 C_{\mathrm{A}} e \pi}{4 \sqrt{2} C_{\mathrm{N}} R}+\frac{(18 e \pi+24 R)}{12 \sqrt{2} C_{\mathrm{N}} e R} k^{\mathrm{M}}+\left(-\frac{2 \sqrt{2}}{3 C_{\mathrm{N}} e}+\frac{\pi}{2 \sqrt{2} C_{\mathrm{N}} R}\right) \mu^{\mathrm{M}}\right]\left(\mathbb{F}+{ }^{\mathrm{T}} \mathbb{F}\right) \\
& +\left[1+\frac{C_{\mathrm{A}} e \pi}{C_{\mathrm{T}} R}+\frac{\left(-24 C_{\mathrm{N}} \pi+9 C_{\mathrm{T}} \pi\right)}{12 C_{\mathrm{N}} C_{\mathrm{T}} R} k^{\mathrm{M}}+\left(-\frac{\pi}{2 C_{\mathrm{N}} R}-\frac{2 \pi}{3 C_{\mathrm{T}} R}\right) \mu^{\mathrm{M}}\right] \mathbb{J}_{t} \\
& +\left[\frac{C_{\mathrm{C}} e \pi+C_{\mathrm{T}} R}{C_{\mathrm{T}} R}-\frac{2 \pi \mu^{\mathrm{M}}}{C_{\mathrm{T}} R}\right] \mathbb{K}_{t} \\
& +\left[1-\frac{C_{\mathrm{A}} e \pi+2 C_{\mathrm{C}} e \pi+4 C_{\mathrm{T}} R}{4 C_{\mathrm{T}} R}+\frac{\left(C_{\mathrm{A}} e \pi+2 C_{\mathrm{C}} e \pi+4 C_{\mathrm{T}} R\right) \mu^{\mathrm{M}}}{2 C_{\mathrm{T}}^{2} e R}\right] \mathbb{K}_{l} .
\end{aligned}
$$

The Hashin-Shtrikman lower bound (22) involves the quantity $\mathbb{C}^{\mathrm{coh}}: \mathbb{T}$ which can be obtained using the formulas (12), (31) and the elementary operations introduced in (26):

$$
\begin{aligned}
\mathbb{C}^{\mathrm{coh}}: \mathbb{T} & =\left[k^{\mathrm{M}}+\frac{4 \mu^{\mathrm{M}}}{3}+\frac{3 k^{\mathrm{M}}-2 \mu^{\mathrm{M}}}{4 R} \pi e\right] \mathbb{E}_{l} \\
& +\sqrt{2}\left[k^{\mathrm{M}}-\frac{2 \mu^{\mathrm{M}}}{3}+\frac{3 k^{\mathrm{M}}+\mu^{\mathrm{M}}}{4 R} \pi e\right] \mathbb{F} \\
& +\left[\frac{C_{\mathrm{A}}\left(-24 k+16 \mu^{\mathrm{M}}\right)}{12 \sqrt{2} C_{\mathrm{T}} R} \pi e+\frac{C_{\mathrm{A}}\left(9 C_{\mathrm{T}} k^{\mathrm{M}}+12 C_{\mathrm{T}} \mu^{\mathrm{M}}\right)}{12 \sqrt{2} C_{\mathrm{N}} C_{\mathrm{T}} R} \pi e\right] \mathrm{T}_{\mathbb{F}} \\
& +\left[\frac{C_{\mathrm{A}} \pi\left(3 k^{\mathrm{M}}-2 \mu^{\mathrm{M}}\right)}{4 C_{\mathrm{N}} R} e-\frac{C_{\mathrm{A}}\left(6 k^{\mathrm{M}} \pi-3 C_{\mathrm{T}} R+2 \pi \mu^{\mathrm{M}}\right)}{3 C_{\mathrm{T}} R} e\right] \mathbb{J}_{t} \\
& +\left[\left(C_{\mathrm{C}}-\frac{2 C_{\mathrm{C}} \pi \mu^{\mathrm{M}}}{C_{\mathrm{T}} R}\right) e\right] \mathbb{K}_{t}+\left[2 \mu^{\mathrm{M}}+\frac{\left(C_{\mathrm{A}}+2 C_{\mathrm{C}}\right) \mu^{\mathrm{M}}}{2 C_{\mathrm{T}} R} \pi e\right] \mathbb{K}_{l} \\
& +o(e)
\end{aligned}
$$

Then, to calculate the expression

$$
\mathbb{C}^{\mathrm{HS}-}=\left[e Z\left\langle\mathbb{C}^{\mathrm{coh}}: \mathbb{T}\right\rangle_{\circlearrowleft}+(1-e Z) \mathbb{C}^{\mathrm{M}}\right]:\left[e Z\langle\mathbb{T}\rangle_{\circlearrowleft}+(1-e Z) \mathbb{I}\right]^{-1},
$$

use is made of the average over all spatial orientations (see (21)) and the inversion relationship (27). Noting that

$$
\begin{cases}\mathbb{E}_{l}:: \mathbb{J}=\frac{1}{3}, & \mathbb{J}_{t}:: \mathbb{J}=\frac{2}{3}, \\ \mathbb{F}:: \mathbb{J}={ }^{\top} \mathbb{F}:: \mathbb{J}=\frac{\sqrt{2}}{3}, & \mathbb{K}_{t}:: \mathbb{J}=\mathbb{K}_{l}:: \mathbb{J}=0, \\ \mathbb{E}_{l}:: \mathbb{K}=\frac{2}{3}, & \mathbb{J}_{t}:: \mathbb{K}=\frac{1}{3}, \\ \mathbb{F}:: \mathbb{K}={ }^{\top} \mathbb{F}:: \mathbb{K}=\frac{-\sqrt{2}}{3}, & \mathbb{K}_{t}:: \mathbb{K}=\mathbb{K}_{l}:: \mathbb{K}=2,\end{cases}
$$

the power series expansion of $\mathbb{C}^{\mathrm{HS}-}$ around the point $e=0$ at the first order reads:

$$
\begin{aligned}
& \mathbb{C}^{\mathrm{HS}-}=\left(\left[3 k^{\mathrm{M}}-2 Z k^{\mathrm{M}} e\right] \mathbb{J}+\left[2 \mu^{\mathrm{M}}-\frac{14}{15} Z \mu^{\mathrm{M}} e\right] \mathbb{K}+o(e)\right): \\
& \left(\left[\frac{C_{\mathrm{N}}}{C_{\mathrm{N}}+k^{\mathrm{M} Z}}+g(e)\right] \mathbb{J}+\left[\frac{15 C_{\mathrm{N}} C_{\mathrm{T}} \mu^{\mathrm{M}}}{15 C_{\mathrm{N}} C_{\mathrm{T}}+12 C_{\mathrm{N}} Z \mu^{\mathrm{M}}+4 C_{\mathrm{T}} Z \mu^{\mathrm{M}}}+h(e)\right] \mathbb{K}+o(e)\right)
\end{aligned}
$$


where

$$
g(e)=\frac{2 C_{\mathrm{N}}^{2} k^{\mathrm{M}} \pi Z}{C_{\mathrm{T}} R\left(C_{\mathrm{N}}+Z k^{\mathrm{M}}\right)^{2}} e+\frac{-9 k^{\mathrm{M}} \pi+2 C_{\mathrm{N}} R}{6 R\left(C_{\mathrm{N}}+Z k^{\mathrm{M}}\right)^{2}} C_{\mathrm{N}} Z e
$$

and

$$
h(e)=\frac{15\left(3 C_{\mathrm{T}}^{2} \pi \mu^{\mathrm{M}}+C_{\mathrm{N}}\left(8 C_{\mathrm{T}}^{2} R-3\left(C_{\mathrm{A}}+2 C_{\mathrm{C}}\right) \pi \mu^{\mathrm{M}}+14 C_{\mathrm{T}} \pi \mu^{\mathrm{M}}\right)\right)}{R\left(4 C_{\mathrm{T}} Z \mu^{\mathrm{M}}+3 C_{\mathrm{N}}\left(5 C_{\mathrm{T}}+4 Z \mu^{\mathrm{M}}\right)\right)^{2}} C_{\mathrm{N}} Z e .
$$

Finally, the passage to the limit $e \rightarrow 0$ allows to derive the analytical expression of the homogeneous Hashin-Shtrikman lower bound:

$$
\mathbb{C}^{\text {hom }}=3 \frac{C_{\mathrm{N}} k^{\mathrm{M}}}{C_{\mathrm{N}}+k^{\mathrm{M} Z}} \mathbb{J}+2 \frac{15 C_{\mathrm{N}} C_{\mathrm{T}} \mu^{\mathrm{M}}}{15 C_{\mathrm{N}} C_{\mathrm{T}}+12 C_{\mathrm{N}} Z \mu^{\mathrm{M}}+4 C_{\mathrm{T}} Z \mu^{\mathrm{M}}} \mathbb{K} .
$$

Hence, the expression of the bulk and shear homogeneous moduli $k^{\text {hom }}$ and $\mu^{\text {hom }}$ are:

$$
\left\{\begin{array}{l}
\frac{k^{\text {hom }}}{k^{\mathrm{M}}}=\frac{\xi_{\mathrm{k}}}{1+\xi_{\mathrm{k}}} \quad \text { with } \quad \xi_{\mathrm{k}}=\frac{C_{\mathrm{N}}}{Z k^{\mathrm{M}}}, \\
\frac{\mu^{\text {hom }}}{\mu^{\mathrm{M}}}=\frac{\xi_{\mu}}{1+\xi_{\mu}} \quad \text { with } \quad \xi_{\mu}=\frac{15}{4\left(1+3 C_{\mathrm{N}} / C_{\mathrm{T}}\right)} \frac{C_{\mathrm{N}}}{Z \mu^{\mathrm{M}}} .
\end{array}\right.
$$

It is worth noting that equations (36) show that a cohesive-volumetric formulation with vanishing tangential cohesive stiffness $\left(C_{\mathrm{T}} \rightarrow 0\right)$ leads to a macroscopic no shear material ( $\mu^{\text {hom }} \rightarrow 0$ ). Moreover, the lower bounds (36) allow to define criteria on the overall loss of stiffnesses, e.g. assuming that a reduction of $5 \%$ on the apparent bulk and shear moduli is admissible:

$$
\left\{\begin{array}{l}
\frac{k^{\text {hom }}}{k^{\mathrm{M}}} \geq 0.95 \text { is ensured for } \xi_{\mathrm{k}} \geq 20 \\
\frac{\mu^{\mathrm{hom}}}{\mu^{\mathrm{M}}} \geq 0.95 \text { is ensured for } \xi_{\mu} \geq 20
\end{array}\right.
$$

Since the geometric hypothesis 1 has no influence for the case of 2-D discretization, it should be mentioned that, for planar isotropic meshes, (36) is a lower bound and (37) is a 'rigorous' criteria. For regular meshes, (36) is an estimate. The accuracy of the obtained results is illustrated in the next section and practical criteria are derived in the section 4.

\section{Numerical experiments}

All calculation presented hereafter were done using the software XPER (Perales et al., 2008, 2010). The analysis is based on embedded linear elastic cohesive-volumetric elements and isotropic medium under plane strain conditions. In Figure 4, the dependency of the overall elastic moduli on the normalto-tangential cohesive stiffness ratio is studied for regular meshes (see Figure 3, left). Bulk material properties are as follows: Young's modulus $E^{\mathrm{M}}=117.5 \mathrm{GPa}$ and Poisson coefficient $\nu^{\mathrm{M}}=0.3$. The evolution of the overall elastic properties as function of the normal cohesive stiffness is illustrated for different normal-to-tangential cohesive stiffness ratios $C_{\mathrm{N}} / C_{\mathrm{T}}$. Numerical experiments show clearly that the overall bulk modulus $k^{\text {hom }}$ is not sensitive to the ratio $C_{\mathrm{N}} / C_{\mathrm{T}}$, as predicted by the theoretical bounds (36). The overall shear modulus $\mu^{\text {hom }}$ decreases with the ratio $C_{\mathrm{N}} / C_{\mathrm{T}}$, and again these results are compatible with the bounds (36). It should be noticed that these bounds are sharper when the normalto-tangential cohesive stiffness ratio decreases. Numerically speaking, the bounds (36) are thus not very accurate for large values of the ratio $C_{\mathrm{N}} / C_{\mathrm{T}}$ as expected.

The results presented in Figure 5 show that the apparent reduction associated to the Young's modulus $E^{\text {hom }} / E^{\mathrm{M}}$ depends on the normal-to-tangential cohesive stiffness ratio as expected by (39). Moreover, we observe that the ratio $C_{\mathrm{N}} / C_{\mathrm{T}}$ leading to an apparent Young's modules reduction $E^{\text {hom }} / E^{\mathrm{M}}$ increases with the Poisson ratio: the dashed lines show that for a material with $\nu^{\mathrm{M}}=0.3$, the Young's modulus 


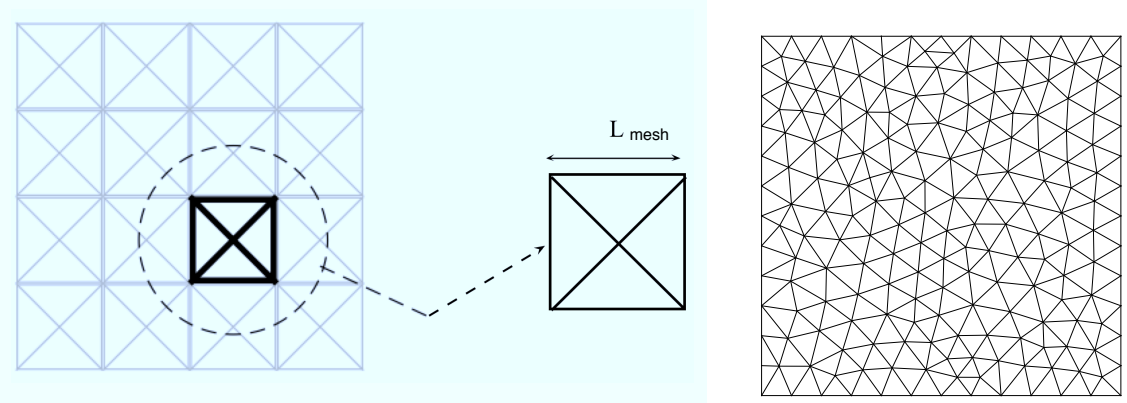

Figure 3: Examples of Regular (left) and Delaunay-type (right) meshes.
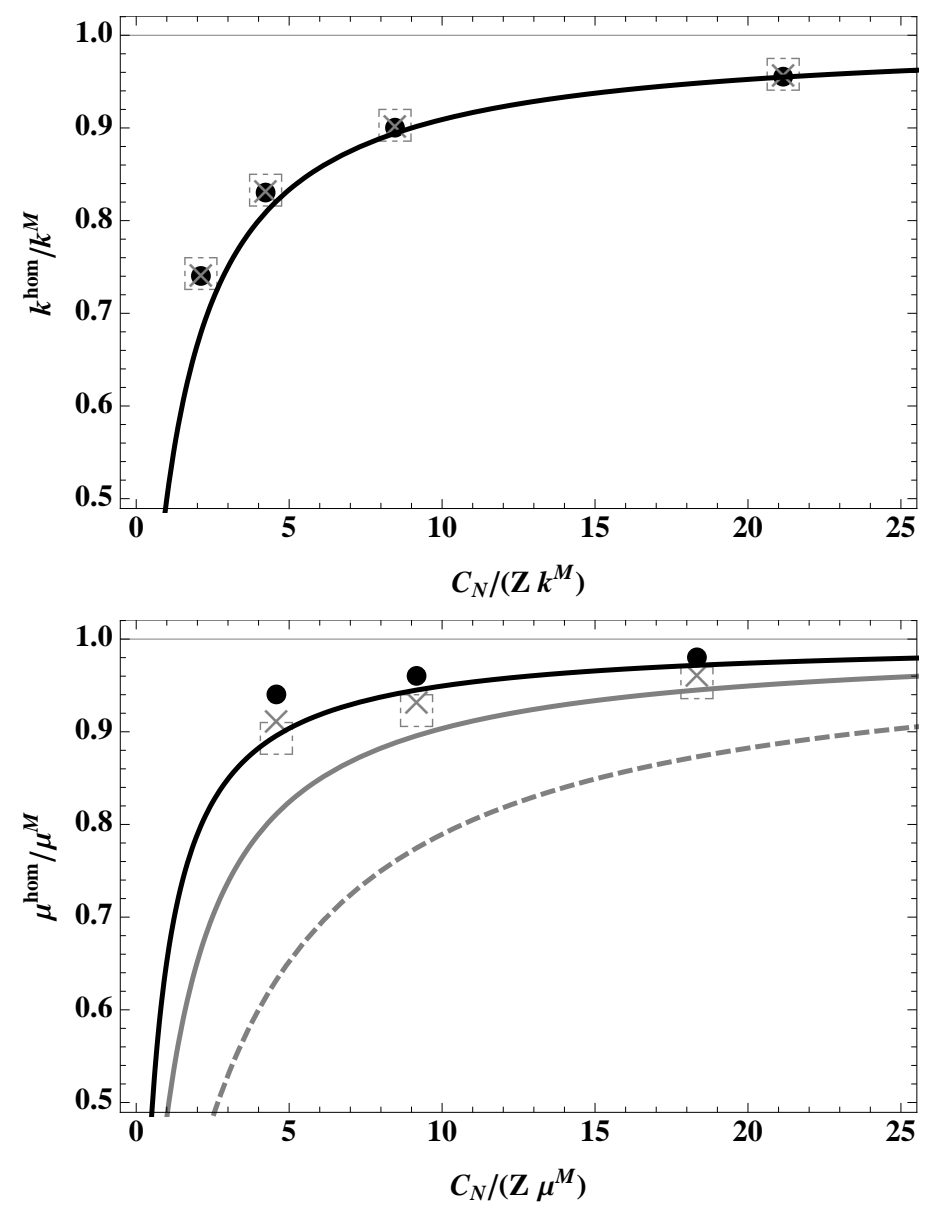

Figure 4: Normalized overall elastic moduli for different cases of the normal-to-tangential cohesive stiffness ratio: bulk modulus (top) and shear modulus (bottom). Numerical results: $C_{\mathrm{N}} / C_{\mathrm{T}}=1 / 3$ black bullets, $C_{\mathrm{N}} / C_{\mathrm{T}}=1$ gray crosses, $C_{\mathrm{N}} / C_{\mathrm{T}}=3$ gray dashed squares. Theoretical bounds (36): $C_{\mathrm{N}} / C_{\mathrm{T}}=1 / 3$ black curve, $C_{\mathrm{N}} / C_{\mathrm{T}}=1$ gray curve, $C_{\mathrm{N}} / C_{\mathrm{T}}=3$ gray dashed curve.

reduction reaches 0.77 when the ratio $C_{\mathrm{N}} / C_{\mathrm{T}}=1$, whereas a material with $\nu^{\mathrm{M}}=0$ needs a ratio $C_{\mathrm{N}} / C_{\mathrm{T}}=1 / 3$ to reach the same reduction; this result is consistent with the proposed criterion (42).

In Figure 6 and Figure 7, the numerical experiments concern both planar Delaunay (closed symbols with variance) and 'cross-triangle quadrilateral' meshes (open symbols) for the case $C_{\mathrm{N}}=C_{\mathrm{T}}$ : as antecedently expected in the previous section, Figure 6 and Figure 7 show that relations (36) are rigorous lower bounds for isotropic meshes (Delaunay) and convenient estimates for regular meshes. Note that these regular meshes exhibit a higher added compliance than isotropic ones. Moreover, these numerical results clearly show that the criteria (37), $\xi_{\mathrm{k}} \geq 20$ and $\xi_{\mu} \geq 20$, ensure that the overall added compliance 


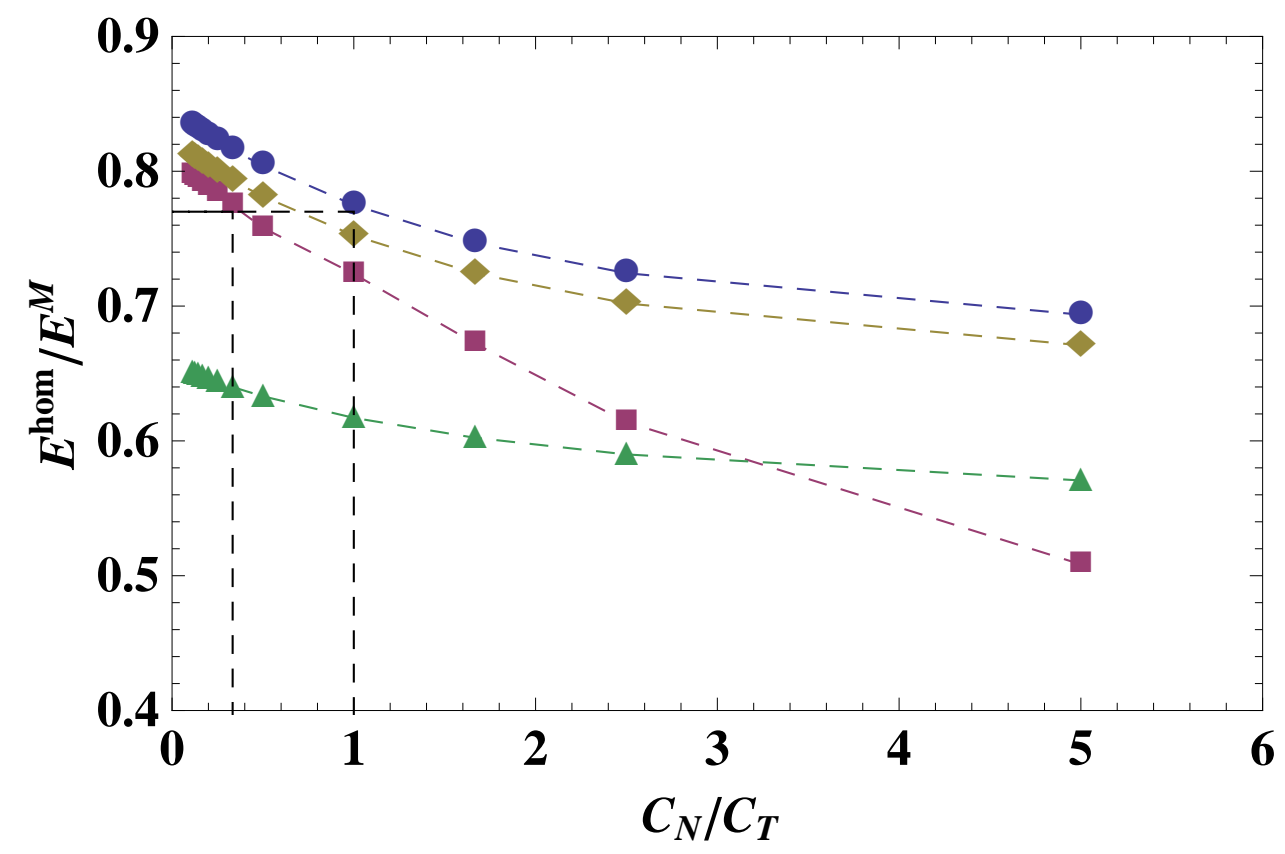

Figure 5: Effect of the tangential-to-normal cohesive stiffness ratio on the overall behaviour: Young apparent reduction vs the ratio $C_{\mathrm{N}} / C_{\mathrm{T}}$ using a square regular mesh $\left(L_{\text {mesh }}=0.05 \mathrm{~mm}\right) . \quad \nu^{\mathrm{M}}=0.3$ (circular symbols), $\nu^{\mathrm{M}}=0.14$ (diamond symbol), $\nu^{\mathrm{M}}=0$ (square symbols) and $\nu^{\mathrm{M}}=-0.7$ (triangular symbols).

do not exceed 5\% (dashed lines in Figure 6 and Figure 7).

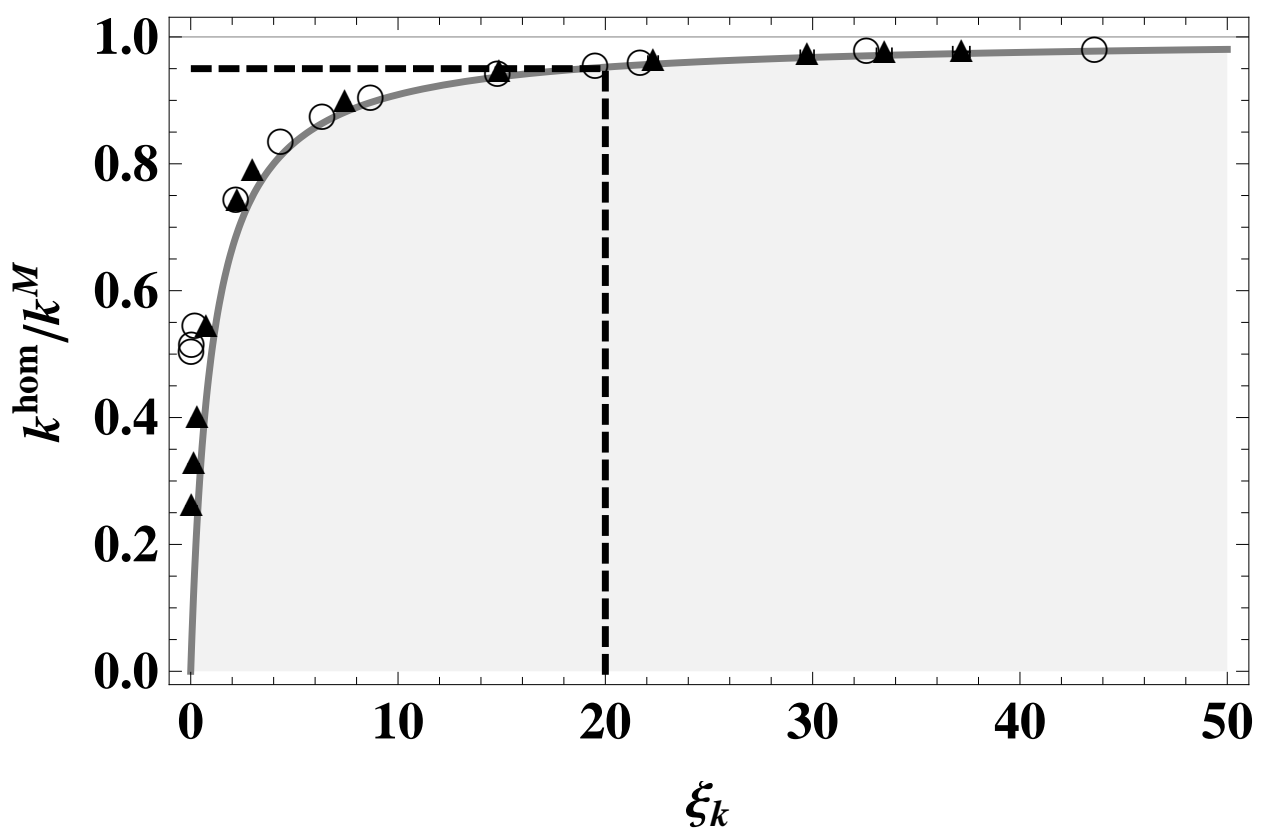

Figure 6: Normalized overall bulk modulus of a cohesive-volumetric formulation with intrinsic CZMs (case $C_{\mathrm{N}}=C_{\mathrm{T}}$ ): lower bounds (36) (thick gray line), criteria (37) (dashed line), numerical results for Delaunay meshes (closed symbols with variance) and for cross-triangle quadrilateral meshes (open symbols). 


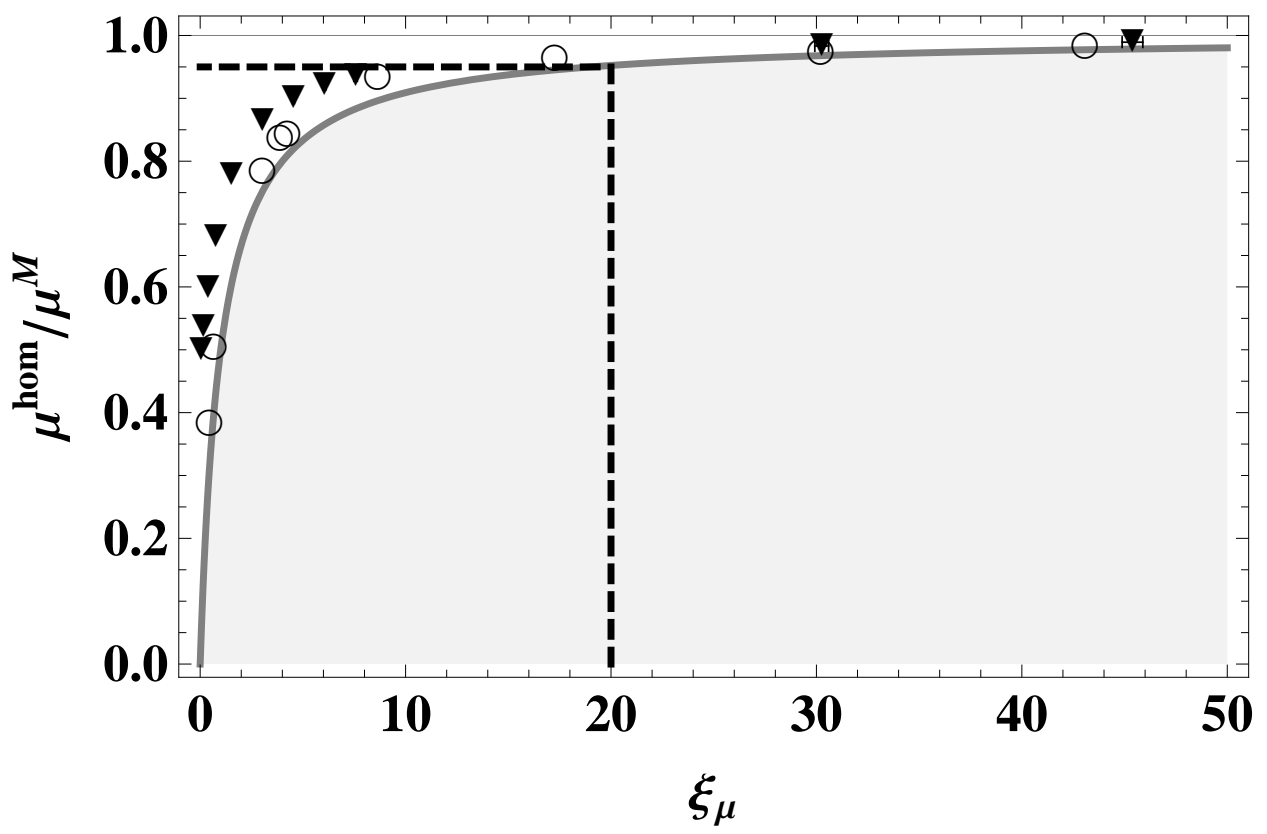

Figure 7: Normalized overall shear modulus of a cohesive-volumetric formulation with intrinsic CZMs (case $C_{\mathrm{N}}=C_{\mathrm{T}}$ ): lower bounds (36) (thick gray line), criteria (37) (dashed line), numerical results for Delaunay meshes (closed symbols with variance) and for cross-triangle quadrilateral meshes (open symbols).

\section{Practical criteria on cohesive stiffnesses}

Criteria on cohesive stiffnesses obtained from micromechanical models have been firstly discussed by Acary and Monerie (2006). Hereafter, use will be made of engineer's moduli (Young moduli and Poisson ratio):

$$
\begin{aligned}
E^{\mathrm{M}} & =\frac{9 k^{\mathrm{M}} \mu^{\mathrm{M}}}{3 k^{\mathrm{M}}+\mu^{\mathrm{M}}} \quad \text { and } \quad \nu^{\mathrm{M}}=\frac{3 k^{\mathrm{M}}-2 \mu^{\mathrm{M}}}{6 k^{\mathrm{M}}+2 \mu^{\mathrm{M}}}, \\
E^{\text {hom }} & =\frac{9 k^{\text {hom }} \mu^{\text {hom }}}{3 k^{\text {hom }}+\mu^{\text {hom }}} \quad \text { and } \quad \nu^{\text {hom }}=\frac{3 k^{\text {hom }}-2 \mu^{\text {hom }}}{6 k^{\text {hom }}+2 \mu^{\text {hom }}} .
\end{aligned}
$$

The additional compliance due to the presence of intrinsic CZMs is measured by two relative apparent reductions $R=E^{\mathrm{hom}} / E^{\mathrm{M}}$ and $r=\nu^{\mathrm{hom}} / \nu^{\mathrm{M}}$, so that cohesive zones do not affect the overall behaviour if, ideally, $R=1$ and $r=1$. Combining (36) and (38) gives for the proposed micromechanical model:

$$
R=\frac{E^{\mathrm{hom}}}{E^{\mathrm{M}}}=\frac{\xi_{\mathrm{E}}}{1+\xi_{\mathrm{E}}} \quad \text { where } \quad \xi_{\mathrm{E}}=\frac{5}{1+(4 / 3)\left(C_{\mathrm{N}} / C_{\mathrm{T}}\right)} \times \frac{C_{\mathrm{N}}}{E^{\mathrm{M} Z}}
$$

and

$$
r=\frac{\nu^{\text {hom }}}{\nu^{\mathrm{M}}}=\frac{15 C_{\mathrm{N}} \nu^{\mathrm{M}}+\left(-1+2 C_{\mathrm{N}} / C_{\mathrm{T}}\right) E^{\mathrm{M}} Z}{15 C_{\mathrm{N}} \nu^{\mathrm{M}}+\left(3+4 C_{\mathrm{N}} / C_{\mathrm{T}}\right) E^{\mathrm{M}} Z \nu^{\mathrm{M}}} .
$$

The equation (39) extends the 1D-equation (3) with a prefactor on $\xi$ and the use of the density $Z$ instead of the mesh size $L_{\text {mesh }}$. This equation shows again that without tangential cohesive stiffness $\left(C_{\mathrm{T}} \rightarrow 0\right)$ an overall no tension material is expected $\left(E^{\text {hom }} \rightarrow 0\right.$ ). Moreover, the situation $R=1$ can not be reached (the overall Young's modulus is always disturbed by the presence of CZMs using a Hashin Shtrikman estimate). However, for a given value of $R \in[0,1$ [, equation (39) leads to an implicit lower bound on the key parameter $C_{\mathrm{N}} /\left(E^{\mathrm{M}} Z\right)$ involving the normal-to-tangential cohesive stiffness ratio $C_{\mathrm{N}} / C_{\mathrm{T}}$ :

$$
\frac{C_{\mathrm{N}}}{E^{\mathrm{M} Z}} \geq \frac{1}{5} \frac{R}{1-R}\left(1+\frac{4}{3} \frac{C_{\mathrm{N}}}{C_{\mathrm{T}}}\right)
$$


Fortunately, reduction $r=1$, i.e. $\nu^{\text {hom }}=\nu^{\mathrm{M}}$, can be reached in (40) fixing the ratio $C_{\mathrm{N}} / C_{\mathrm{T}}$ and thus defining 'invisible' intrinsic CZMs for the Poisson ratio:

$$
\nu^{\text {hom }}=\nu^{\mathrm{M}} \Rightarrow \frac{C_{\mathrm{N}}}{C_{\mathrm{T}}}=\frac{1}{2} \frac{1+3 \nu^{\mathrm{M}}}{1-2 \nu^{\mathrm{M}}}
$$

It is worth noting that the condition (42) ensures same elastic reduction for bulk, shear and Young moduli:

$$
\frac{k^{\mathrm{hom}}}{k^{\mathrm{M}}}=\frac{\mu^{\mathrm{hom}}}{\mu^{\mathrm{M}}}=\frac{E^{\mathrm{hom}}}{E^{\mathrm{M}}} .
$$

We underline that relationships (43) were proposed by Li and Wang (2004) as a closure condition in their self-consistent estimate for pure hydrostatic loadings. The present approach can thus be seen as an improvement of the results of Li and Wang (2004) to arbitrary type of loadings.

As before mentioned, the cohesive interface density $Z$ corresponds to the 'specific' interface surface: $Z=\mathcal{A} / \mathcal{S}$ where $\mathcal{A}$ is the total edge length (resp. area) in 2-D (resp. in 3-D) and $\mathcal{S}$ is the total area (resp. volume) of the 2-D (resp. 3-D) meshed body. For any discretization characterized by a mesh size $L_{\text {mesh }}, \mathcal{A}$ is proportional to $L_{\text {mesh }}^{q-1}$ of the mesh and $\mathcal{S}$ is proportional to $L_{\text {mesh }}^{q}$ where $q$ is the considered dimension; density $Z$ is thus inversely proportional to the mesh size and can be expressed as:

$$
Z=\frac{\gamma}{L_{\text {mesh }}}
$$

where the real $\gamma$ depends on the spatial distribution of the considered mesh. Hence, relations (41), (42) and (44) allow to define a practical criterion on the ratio $C_{\mathrm{N}} L_{\text {mesh }} / E^{\mathrm{M}}$ extending semi-empirical criteria previously proposed in the literature (Espinosa and Zavattieri, 2003; Tomar et al., 2004; Turon et al., 2007):

$$
\frac{C_{\mathrm{N}} L_{\mathrm{mesh}}}{E^{\mathrm{M}}} \geq \gamma \frac{R}{1-R} \frac{1}{3\left(1-2 \nu^{\mathrm{M}}\right)} .
$$

Moreover, the intermediate criteria (41) and (42) together with the apparent reductions $R<1$ and $r=1$ allow to ensure accurate estimates of the elastic wave speeds, in fact better than of the apparent compliance. The apparent dilatational and shear wave speeds are given by $c_{d}^{\text {hom }}=\sqrt{\left(\lambda^{\text {hom }}+2 \mu^{\text {hom }}\right) / \rho^{\text {hom }}}$ and $c_{s}^{\text {hom }}=\sqrt{\mu^{\text {hom }} / \rho^{\text {hom }}}$ respectively, where $\lambda^{\text {hom }}$ is the effective Lame's coefficient and $\rho^{\text {hom }}$ is the effective density of the cohesive-volumetric medium. Assuming that the finite element discretization does not affect the density $\rho^{\text {hom }}=\rho^{\mathrm{M}}\left(\rho^{\mathrm{M}}\right.$ being the density of the matrix phase) and since the criterion (42) ensures that the reduction $r=1$ is reached for the Poisson ratio, the apparent reductions of the elastic wave speeds read:

$$
\begin{gathered}
\frac{c_{d}^{\text {hom }}}{c_{d}^{\mathrm{M}}}=\sqrt{\frac{\lambda^{\text {hom }}+2 \mu^{\text {hom }}}{\lambda^{\mathrm{M}}+2 \mu^{\mathrm{M}}}}=\sqrt{\frac{E^{\text {hom }}}{E^{\mathrm{M}}}}=\sqrt{R}, \\
\frac{c_{s}^{\text {hom }}}{c_{s}^{\mathrm{M}}}=\sqrt{\frac{\mu^{\mathrm{hom}}}{\mu^{\mathrm{M}}}}=\sqrt{\frac{E^{\text {hom }}}{E^{\mathrm{M}}}}=\sqrt{R} .
\end{gathered}
$$

Thus, tolerating for example a reduction of $5 \%$ for the Young's modulus, i.e. $R=0.95$, leads to a reduction lower than $3 \%$ for the elastic wave speeds $(\sqrt{R} \simeq 0.97)$.

The application of the previous criterion (45) to the case of planar meshes is studied hereafter where the influence of the discretization morphology is illustrated.

\section{Application to planar meshes}

\subsection{Regular meshes}

We consider the case of a '2-D cross-triangle quadrilateral' meshes: each element square is divided into four isosceles triangles as shown in Figure 3 (a). Note that for such case of mesh geometry, the property 
of the orientational equi-probability of inclusions is not respected since the mesh is not really isotropic. The obtained criterion (36) should be thus understood as an estimate and not as a lower bound. The parameter $\gamma$ is equal in that case to $\gamma=2(1+\sqrt{2})=4.83$ (see Appendix A), this result combined with the relation (42) and the inequality (45) provides a ' 2 -D practical $5 \%$ criteria' $(R=0.95)$ for regular meshes:

$$
\frac{C_{\mathrm{N}} L_{\text {mesh }}}{E^{\mathrm{M}}} \geq \frac{30}{1-2 \nu^{\mathrm{M}}} \quad \text { and } \quad \frac{C_{\mathrm{T}}}{C_{\mathrm{N}}}=2 \frac{1-2 \nu^{\mathrm{M}}}{1+3 \nu^{\mathrm{M}}}
$$

For a bulk material with $\nu^{\mathrm{M}}=0.2$, these criteria give $C_{\mathrm{T}}=0.75 C_{\mathrm{N}}$ and $C_{\mathrm{N}} \geq 50 E^{\mathrm{M}} / L_{\text {mesh }}$, which corresponds to the numerical criterion proposed by Turon et al. (2007). Conversely, combining (42) and (45) shows that, for a bulk material with $\nu^{\mathrm{M}}=0.2$, the criterion proposed by Espinosa and Zavattieri (2003), $\frac{C_{\mathrm{N}} L_{\text {mesh }}}{E^{\mathrm{M}}} \geq 10$, corresponds to a reduction $R=0.78$, which guaranties only that the loss in apparent Young's modulus is less than about $22 \%$.

\subsection{Delaunay meshes}

In Delaunay-type discretizations (see Figure 3 (b)), the length of an edge has a probability density function depending on the density of the underlying node-point process (Stoyan et al., 1995; Muche, 1996). The parameter $\gamma$ is thus a stochastical parameter given by a mean and a variance. For a chosen mesh generation, a node-point density is obtained and the variance of $Z$ can be computed. The mean value of $Z$ can be estimated using statistical results of Muche (1996) and introducing a reference edge length (corresponding to an equivalent equilateral triangle):

$$
Z=\frac{\gamma}{L_{\text {mesh }}} \quad \text { where } \quad \gamma=2.97
$$

This result combined with the relation (42) and the inequality (45) provides a ' $2-D$ practical $5 \%$ criteria' $(R=0.95)$ for Delaunay meshes:

$$
\frac{C_{\mathrm{N}} L_{\mathrm{mesh}}}{E^{\mathrm{M}}} \geq \frac{19}{1-2 \nu^{\mathrm{M}}} \quad \text { and } \quad \frac{C_{\mathrm{T}}}{C_{\mathrm{N}}}=2 \frac{1-2 \nu^{\mathrm{M}}}{1+3 \nu^{\mathrm{M}}}
$$

The result obtained by application of the criteria (48) and (50) is plotted in Figure 8 where the elastic energy of a medium containing embedded CZMs is compared to the elastic energy of a medium without CZMs (standard finite element method). The obtained results show that both regular and Delaunay meshes induce a global loss of elastic energy that does not exceed $5 \%$ when the cohesive stiffnesses are suitably calibrated following (48) and (50). When the density $Z$ is fixed at the same value, the difference between the two types of meshes is not significant as shown in Figure 8. This result validates the fact that the overall behaviour obtained with the Hashin-Shtrikman scheme (36) is a convenient estimate even if the property of isotropic meshes is not respected.

\section{Concluding remarks}

The overall constitutive behaviour of an elastic medium with embedded cohesive inclusions has been studied. An equivalent 'matrix-inclusions' composite is considered as a representation of a cohesivevolumetric finite element modelling. As result of this micromechanical model, the following points can be highlighted:

- Rigorous lower bounds on the normal, $C_{\mathrm{N}}$, and the tangential, $C_{\mathrm{T}}$, cohesive stiffness have been obtained for isotropic planar meshes as well as convenient criteria for regular meshes. The practical criteria are given as function of bulk material properties $\left(E^{\mathrm{M}}, \nu^{\mathrm{M}}\right)$ for a given mesh density $Z$ (mesh size $L_{\text {mesh }}$ and mesh morphology parameter $\gamma$ ). 


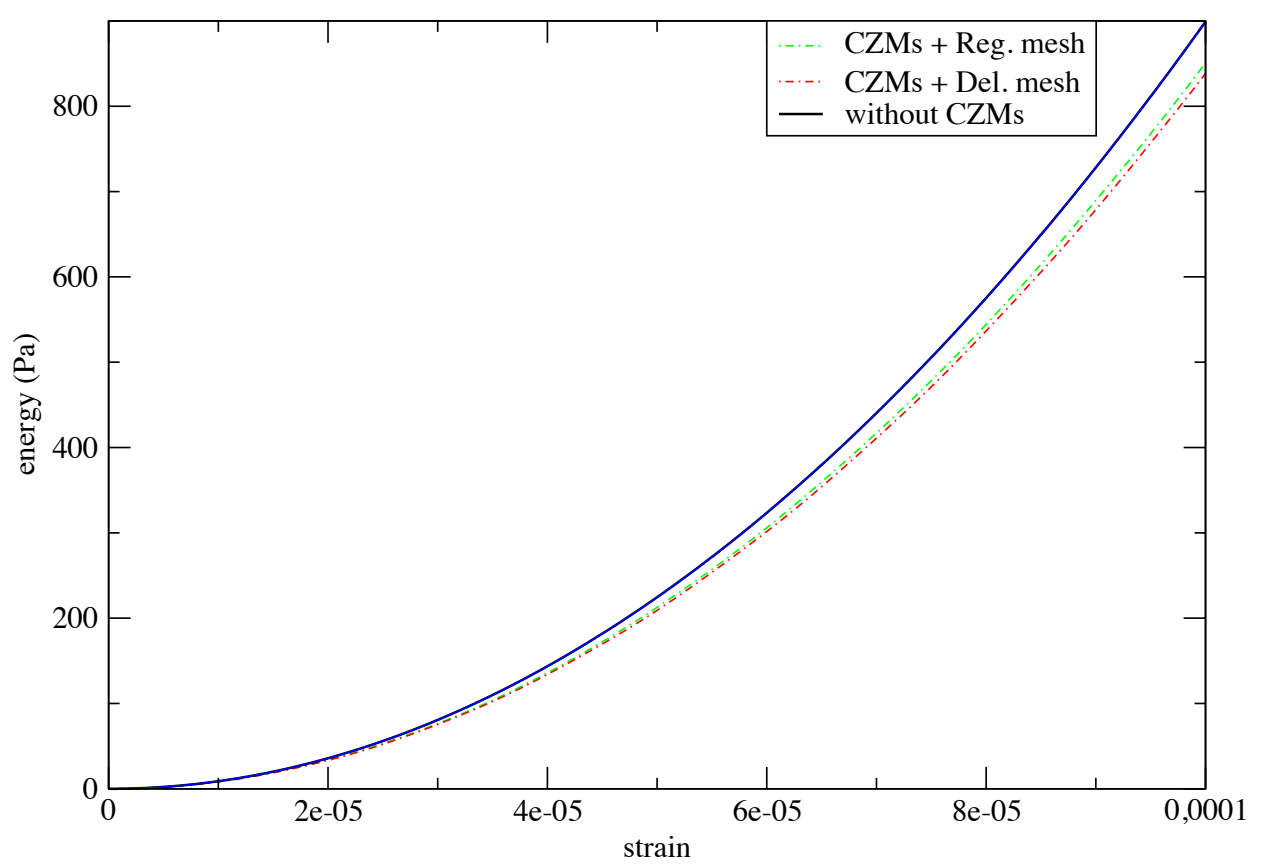

Figure 8: Overall elastic energy (area under the stress-strain curve) of a square cell during a shear test: standard finite element modeling without CZMs (continuous line), cohesive-volumetric finite element modeling using a Delaunay mesh (lower dot-dashed curve) or using a Regular mesh (upper dot-dashed curve).

- Intermediate results of this study show that a cohesive-volumetric approach with a vanishing tangential cohesive stiffness $\left(C_{\mathrm{T}} \rightarrow 0\right)$ leads to an overall no shear material.

- The accuracy of these results has been numerically tested for both Delaunay and regular meshes. The numerical part shows a consistence between numerical results and analytical analysis.

\section{Acknowledgements}

The authors are indebted to Dr. M. Bornert for useful suggestions in orientational averaging and to Dr. F. Perales for providing XPER software facilities.

\section{A Expression of the cohesive density for the case of 2-D regular meshes}

We consider a square cell meshed with regular squares divided into four isosceles triangles. We denote by $N_{t}$ the number of the triangular elements, $L_{\text {mesh }}$ the length of the larger side of each triangle and $L_{\text {tot }}$ the total length of the boundaries between the underlying meshes (total length of the cohesive interfaces). Associating to the cohesive zones a fictitious thickness $e$, the volume fraction $f=e Z$ of cohesive interfaces reads:

$$
f=\frac{e L_{\text {tot }}}{e L_{\text {tot }}+N_{t}\left(L_{\text {mesh }}^{2} / 4\right)},
$$

hence, the expression of the density parameter $Z$ is asymptotically obtained $(e \rightarrow 0)$ :

$$
Z=\frac{L_{\text {tot }}}{N_{t}\left(L_{\text {mesh }}^{2} / 4\right)} .
$$

The total cohesive length $L_{\text {tot }}$ is linked to the mesh length $L_{\text {mesh }}$ and the triangles number $N_{t}$ by the relationship:

$$
L_{\text {tot }}=2(\sqrt{2}+1) \frac{N_{t}}{4} L_{\text {mesh }}+\sqrt{N_{t}} L_{\text {mesh }},
$$


which allows deriving an approximation of $Z$ for large number of mesh elements $\left(N_{t} \rightarrow \infty\right)$ :

$$
Z=\frac{\gamma}{L_{\text {mesh }}} \quad \text { with } \quad \gamma=2(1+\sqrt{2}) .
$$

\section{References}

D. Dugdale, Yielding of steel sheets containing slits, Journal of Mechanics and Physics of Solids 8 (1960) 100-104.

G. I. Barenblatt, The mathematical theory of equilibrium cracks in brittle fractures, Advances In Applied Mechanics 7 (1962) 55-129.

M. Tijssens, L. Sluys, E. van der Giessen, Numerical simulation of quasi-brittle fracture using damaging cohesive surfaces, European Journal of Mechanics A/Solids 19 (2000) 761-779.

A. Turon, C. Dâvila, P. Camanho, J. Costa, An engineering solution for mesh size effects in the simulation of delamination using cohesive zone models, Engineering Fracture Mechanics 4 (2007) 16651682 .

H. Espinosa, P. Zavattieri, A grain level model for the study of failure initiation and evolution in polycrystalline brittle materials. Part I: Theory and numerical implementation, Mechanics of Materials 35 (2003) $333-364$.

V. Tomar, J. Zhai, M. Zhou, Bounds for element size in a variable stiffness cohesive finite element model, International Journal for Numerical Methods in Engineering 61 (2004) 1894-1920.

E. Benvenuti, A regularized XFEM framework for embedded cohesive interfaces, Computer Methods in Applied Mechanics and Engineering 197 (2008) 4367-4378.

T.S. Hille, A.S.J. Suiker, S. Turteltaub, Microcrack nucleation in thermal barrier coating systems, Engineering Fracture Mechanics 76 (2009) 813-825.

N. Blal, L. Daridon, Y. Monerie, S. Pagano, Criteria on the artificial compliance inherent to the intrinsic cohesive zone, Comptes Rendus de Mécanique 339 (2011) 789-795.

V. Acary, Y. Monerie, Nonsmooth fracture dynamics using a cohesive zone approach, Technical Report RR-6032, Institut National de Recherche en Informatique et en Automatique, 2006.

J.-C. Michel, P. Suquet, F. Thébaud, Une modélisation du rôle des interfaces dans le comportement des composites à matrice métallique., Revue Européenne des Eléments Finis 4 (1994) 573-595.

Z. Hashin, S. Shtrikman, A variational approach to the theory of the elastic behaviour of multiphase materials, Journal of the Mechanics and Physics of Solids 11 (1963) 127-140.

J. R. Willis, Bounds and self-consistent estimates for the overall properties of anisotropic composites, Journal of the Mechanics and Physics of Solids 25 (1977) 185-202.

J.-M. Gatt, Y. Monerie, D. Laux, D. Baron, Elastic behavior of porous ceramics: application to nuclear fuel materials, Journal of Nuclear Materials 336 (2005) $145-155$.

M. Bornert, T. Berthau, P. Gilormini (Eds.), Homgénéisation en mécanique des matériaux 2 - Comportements non linéaires et problèmes ouverts, Hermès, 2001.

I. Sevostianov, N. Yilmaz, V. Kushch, V. Levin, Effective elastic properties of matrix composites with transversely-isotropic phases, International journal of solids and structures 42 (2005) 455-476. 
F. Perales, S. Bourgeois, A. Chrysochoos, Y. Monerie, Two field multibody method for periodic homogenization in fracture mechanics of nonlinear heterogeneous materials, Engineering Fracture Mechanics 75 (2008) 3378-3398.

F. Perales, F. Dubois, Y. Monerie, B. Piar, L. Stainier, Multi-body nscd strategy as a multi-domain solver. Application to code coupling dedicated to the modeling of fracture of heterogeneous media, European Journal of Computational Mechanics 19 (2010) 389-417.

S. Li, G. Wang, On damage theory of a cohesive medium, International Journal of Engineering Science 42 (2004) 861-885.

D. Stoyan, W. Kendall, J. Mecke, Stochastic geometry and its applications, John Wiley and Sons, Chichester, New York, Brisbane, Toronto, Singapore, 1995.

L. Muche, Distributional properties of the three-dimensional Poisson Delaunay cell, Journal of statistical physics 84 (1996) 147-167. 